\title{
COSMIC RAY HITS IN THE CENTRAL NERVOUS SYSTEM AT SOLAR MAXIMUM
}

\author{
S. B. Curtis (1), M. E. Vazquez (2), J. W. Wilson (3) and M.-H. Y. Kim (3) \\ (1) Fred Hutchinson Cancer Research Center, Seattle, WA 98104, USA \\ (2) Brookhaven National Laboratory, Upton Long Island, New York, NY 11973, USA \\ (3) NASA Langley Research Center, Hampton VA 23681-0001, USA
}

\begin{abstract}
It has been suggested that a manned mission to Mars be launched at solar maximum rather than at solar minimum to minimize the radiation exposure to galactic cosmic rays. It is true that the number of hits from highly ionizing particles to critical regions in the brain will be less at solar maximum, and it is of some interest to estimate how much less. We present here calculations for several sites within the brain from iron ions $(z=$ 26) and from particles with charge, $z$, greater than or equal to 15 . The same shielding configurations and sites in the brain used in an earlier paper for solar minimum are employed so that direct comparison of results between the two solar activity conditions can be made. A simple pressure-vessel wall and an equipment room onboard a spacecraft are chosen as shielding examples. In the equipment room, typical results for the thalamus $\left(100 \mu \mathrm{m}^{2}\right.$ area) are that the probability of any given cell nucleus being hit decreases from 10 percent at solar minimum to 6 percent at solar maximum for particles with $z$ greater than or equal to 15 and from 2.3 percent to 1.3 percent for iron ions. We conclude that this modest decrease in hit frequency (less than a factor of two) is not a compelling reason to avoid solar minimum for a manned mission to Mars.
\end{abstract}

\section{INTRODUCTION}

A common assumption in estimating radiation risk on a manned Mars mission has been that it would occur in the three-year interval surrounding the period of minimum solar activity ("solar minimum") in order to minimize the probability that a large solar particle event would be encountered by the crew in flight. This meant, however, that maximum intensities of galactic cosmic rays would be encountered, since the magnetic shielding provided by the solar magnetic field and solar wind is at a minimum during this time. Thus, there has been interest in estimating the risk from the galactic cosmic ray environment as it presents itself during the quiet period of the eleven-year solar cycle. It has been shown that every second minimum over the last few solar cycles has resulted in similar intensities, and a "standard" maximum galactic cosmic ray spectrum has been developed for the "worst case" scenerio which was found and well-measured in 1977.

Recently, it was suggested that a manned mission to Mars might be initiated in the period of solar maximum conditions, that is, when the galactic cosmic ray intensities are at a minimum. At least part of the rationale for this is that although the probability for encountering a large solar particle event increases, the intensities of the galactic cosmic rays, for which the risks still have large uncertainties, would be minimized. It could be argued that no matter when the mission is launched, a "safe haven" for the crew should be designed into the spacecraft for use during the advent of a large solar particle event. This reasoning suggests that it is important to assess the effect of using solar maximum rather than solar minimum input spectra in risk estimations. It is particularly important for those situations in which the most important element of the risk is hypothesized to come from the heavy component of the galactic cosmic rays. This is the case for the yet unknown but potentially important risk to the central nervous system from infrequent but very highly ionizing high-energy charged particles (Curtis, 1973, Grahn, 1973, Gauger et al., 1986, NCRP, 1989).

\section{PREVIOUS RESULTS FOR SOLAR MINIMUM SPECTRA}

In a previous paper, we have presented calculations of the estimated number of direct hits and the percentage of cells directly hit in several critical organs of the central nervous system (CNS) by components of the galactic cosmic rays on space missions outside the geomagnetosphere at solar minimum (Curtis et al., 1998). Nuclear interactions within the self-shielding provided by the body itself and within two shielding configurations typical of a manned mission to Mars were included in the calculation. The CNS regions chosen were (1) the macula, the central region of the retina; (2) a point located in the genu, the confluence of the anterior and posterior limbs of the internal capsule (selected because of its proximity with the basal ganglia and the thalamus, two critical brain nuclei); (3) the nucleus basalis of Meynert; (4) the nearby hippocampus. The results showed: 
1. For a three-year mission to Mars, assuming a 1977 spectrum of galactic cosmic rays, the percentage of cells hit at least once depended linearly on the cross-sectional area assumed for the "critical" area of the cells, as well as on the shielding configuration. Values varied from $1 \%$ [midget foveal ganglion cell nuclei $\left(40 \mathrm{\mu m}^{2}\right.$ area) hit by iron ions (charge $z=26$ ) in the "equipment room" shielding configuration, defined below] to $46 \%$ [brain neural cells $\left(471 \mathrm{\mu m}^{2}\right.$ area) hit by particles with charge $z$ equal to or greater than 15 in the "pressure vessel" configuration, also defined below].

2. Roughly 6 million out of some 43 million hippocampal cells and 55 thousand out of 1.8 million thalamus cell nuclei would be directly hit by one or more iron ions $(z=26)$ on such a mission for space travelers inside the "pressure vessel". Also, roughly 20 million out of 43 million hippocampal cells and 230 thousand out of 1.8 million thalamus cell nuclei would be directly hit by one or more particles with $z$ greater than or equal to 15 on such a mission. Other figures were presented for the other critical sites.

It was concluded that the number of cells hit by such highly ionizing particles is not insignificant and that it is important to establish whether a single nuclear hit is sufficient for neuronal inactivation or if a hit in some cytoplasmic domain might elicit an effect potentially initiating a cascade of events leading to cell killing or functional impairment.

\section{PRESENT CALCULATIONS}

For the calculations presented here, only the input spectra of the galactic cosmic rays were changed, keeping all other variables constant, such as shielding, position and size of the cells considered, and mission duration. Thus a direct comparison can be made with the previous results, so that the effect of changing only the time of mission launch (from solar minimum to solar maximum) can be directly assessed. Justification for selection of the above sites are given in the previous paper (Curtis et al., 1998). The sites selected represent regions controlling important functions of vision (macula), motor behavior, balance, ordering and sequencing behavioral patterns (basal ganglia), transition from sleep to the alert state (thalamus), learning and memory (hippocampus) and complex cognitive and affective behaviors (nucleus basilis of Meynert).

The four sites in the brain (basal ganglia, thalamus, hippocampus and nucleus basalis of Meynert) were considered to be adequately represented by a single point in the genu. Small variations in location within the brain do not alter significantly the fluences of particles arriving there. The locations of the resulting two points chosen for the calculations (the macula and the point in the genu) were determined by MRI scans and double-checked with a standard Brain Atlas. The astronaut geometry is represented by the Computerized Anatomical Man (CAM) model (Billings and Yucker, 1973). The CAM is an extremely detailed geometrical representation of the human anatomy for use in investigations dealing with exposures of astronauts to the natural space radiation environment. A region is defined by (1) its material composition, (2) a list of surfaces which bound the region, and (3) the coordinates of a point inside the region. The shield distributions were generated using an equi-solid-angle ray-tracing technique to compute 512 rays (thicknesses through all the materials encountered) over a solid angle of $4 \pi$ steradians. Figures 1 and 2 show cross-sections of the CAM head with the retina (macula) and brain postions (genu) indicated.
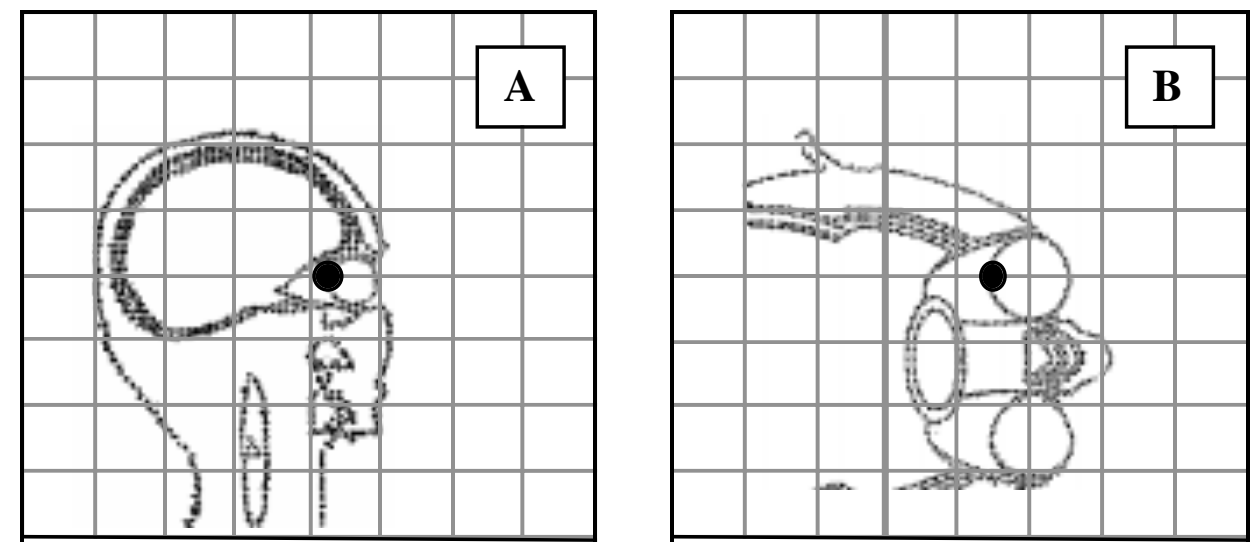

Fig. 1. Retina (macula) location in the CAM model head (black dot). A, Sagital section: the location is $8.39 \mathrm{~cm}$ from the top of the scull. Scale: $5.08 \mathrm{~cm}$ grid. B, Transaxial partial section: the lateral distance to the edge of the scull is $3.19 \mathrm{~cm}$. Scale $2.54 \mathrm{~cm}$ grid. 

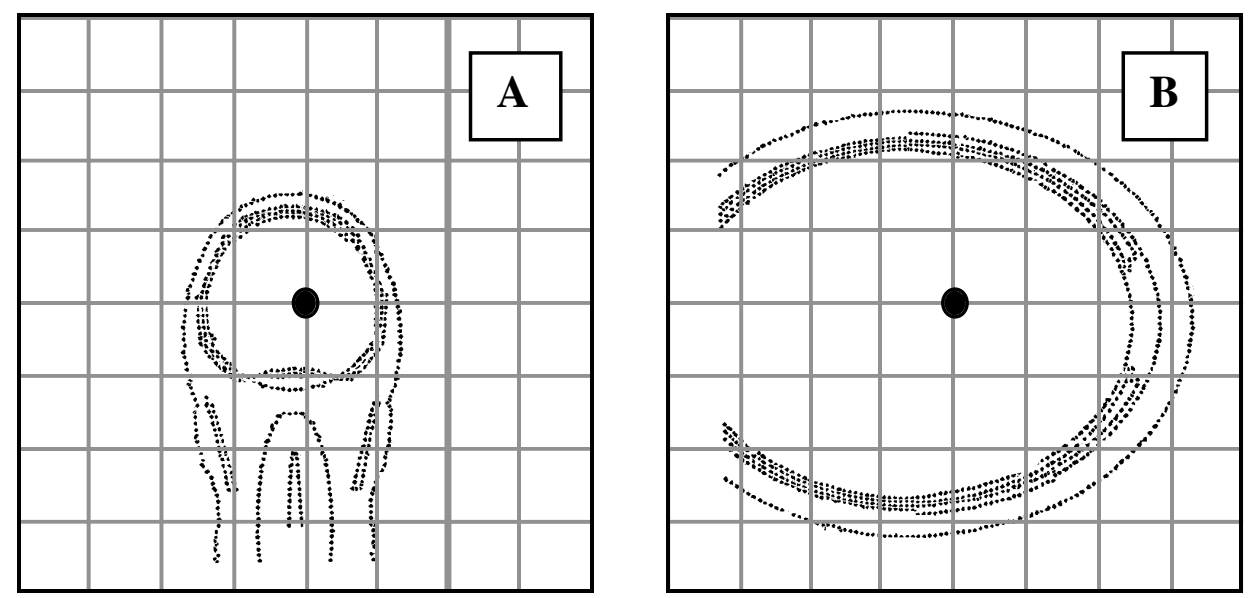

Fig. 2 Brain location in the CAM model head (black dot) of the interior point (average location of the genu, hippocampus, thalamus, and nucleus basalis of Meynert). A, Coronal section: the location is $7.8 \mathrm{~cm}$ from the top of the scull. Scale: $5.08 \mathrm{~cm}$ grid. B, Transaxial section: the lateral distance to the edge of the scull is $6.6 \mathrm{~cm}$ and the distance to the front of the scull is $8.14 \mathrm{~cm}$. Scale: $2.54 \mathrm{~cm}$ grid.

The spacecraft geometry is simplified in the present calculation since no specific mission is targeted. The main living space of the vehicle (which is designated the "pressure vessel") is considered to be a wall and micrometeroid bumper represented by a sphere of thickness $1 \mathrm{~g} / \mathrm{cm}^{2}$ aluminum. The "equipment room" is the pressure vessel augmented with equipment racks which are assumed to add $5 \mathrm{~g} / \mathrm{cm}^{2}$ aluminum-equivalent thickness (Wilson and Denn, 1976). The primary and secondary particle fluence rates within the astronaut were generated within the CAM model by solving the Boltzmann equation using the HZETRN system (Wilson et al., 1995, Cucinotta et al., 1995) using a 1970 Solar Maximum Environmental Model isotropically incident on the spacecraft.

The mass distributions (from 512 rays at each position) were used to generate fluence rates of the various components of galactic cosmic rays (and their secondaries) at the two positions of interest over a three-year journey of the spacecraft outside the geomagnetosphere. The galactic cosmic ray spectra were assumed to be constant throughout the entire round-trip journey to Mars. The fluence rates were multiplied by the mission duration (3 years) and the cross-sectional area of interest to obtain the average number of hits. Fluence rates were assumed constant over the critical regions selected. Assuming Poisson statistics, the probability of one or more hits by particles with charge equal or greater than $z$ is one minus the probability of no hits by such particles; the latter, in turn, is $\exp (-n)$, where $n$ is the sum of the average hits from all particles with charge greater than or equal to $z$ at the point of interest.

\section{RESULTS}

The number of hits from the various components of the galactic cosmic rays (plus nuclear secondaries) to a cross-sectional area of $180 \mu \mathrm{m}^{2}$ corresponding to a midget foveal ganglion cell in the macula of the retina within the pressure vessel over a three-year time span is shown as the thick line in Figure 3 . The thin line in the figure shows the results from the solar minimum calculation for comparison. The number of hits of a given particle type with charge $z$ is plotted against $z$. We call these "direct" hits since the center of the track (i.e., the portion of greatest ionization density) goes through the area. In this calculation, we neglect "glancing" hits where only the delta rays from the track hit the area. Although they may cause some damage, we neglect them here because the damage will be of a "low-LET" nature and the probability of their modifying function is assumed to first approximation to be negligible.

Probabilities of one or more hits by particles with charge equal to or greater than $z$ plotted against $z$ for a cross sectional area of $100 \mu \mathrm{m}^{2}$ within the brain is shown in Figure 4 for both solar maximum and solar minimum conditions inside the equipment room on a three-year Mars mission. For all charged components, the difference between the results for solar minimum and solar maximum is less than a factor of two.

With knowledge of the cross sectional areas of presumed sensitive regions of the various cells within the critical sites, the direct hit probabilities at solar maximum can be calculated for the two shielding configurations and compared with the previous calculations for solar minimum conditions. The results are given in Table 1. 


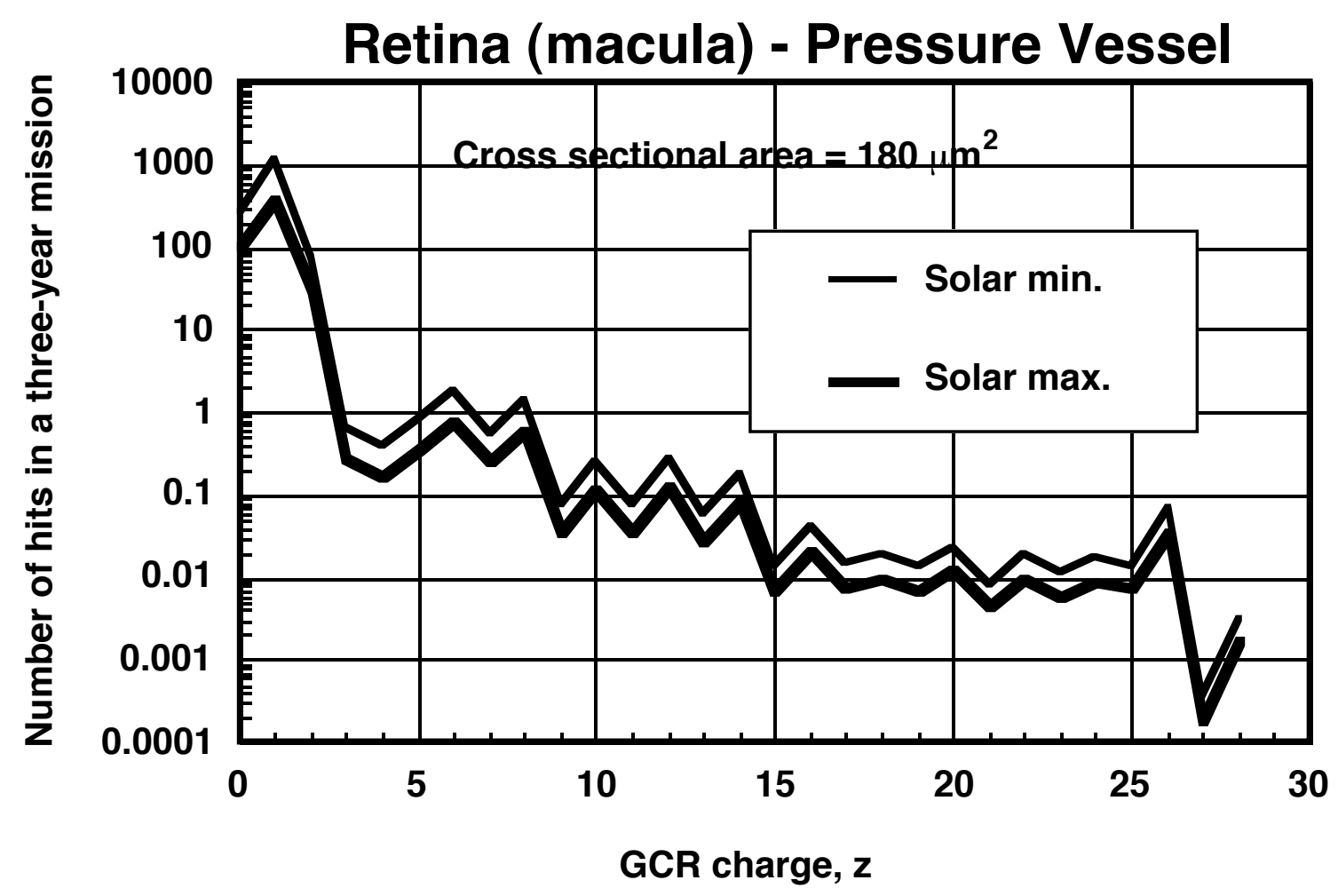

Fig. 3. The average number of direct hits from components of the 1970-spectrum (solar max, thick line) and the 1977-spectrum (solar min, thin line) of galactic cosmic rays plotted as a function of charge $z$ to a site of cross sectional area $180 \mu \mathrm{m}^{2}$ at the position of the macula of the retina in the CAM model of the head of a space traveler in a pressure vessel of a spacecraft on a three-year mission to Mars.

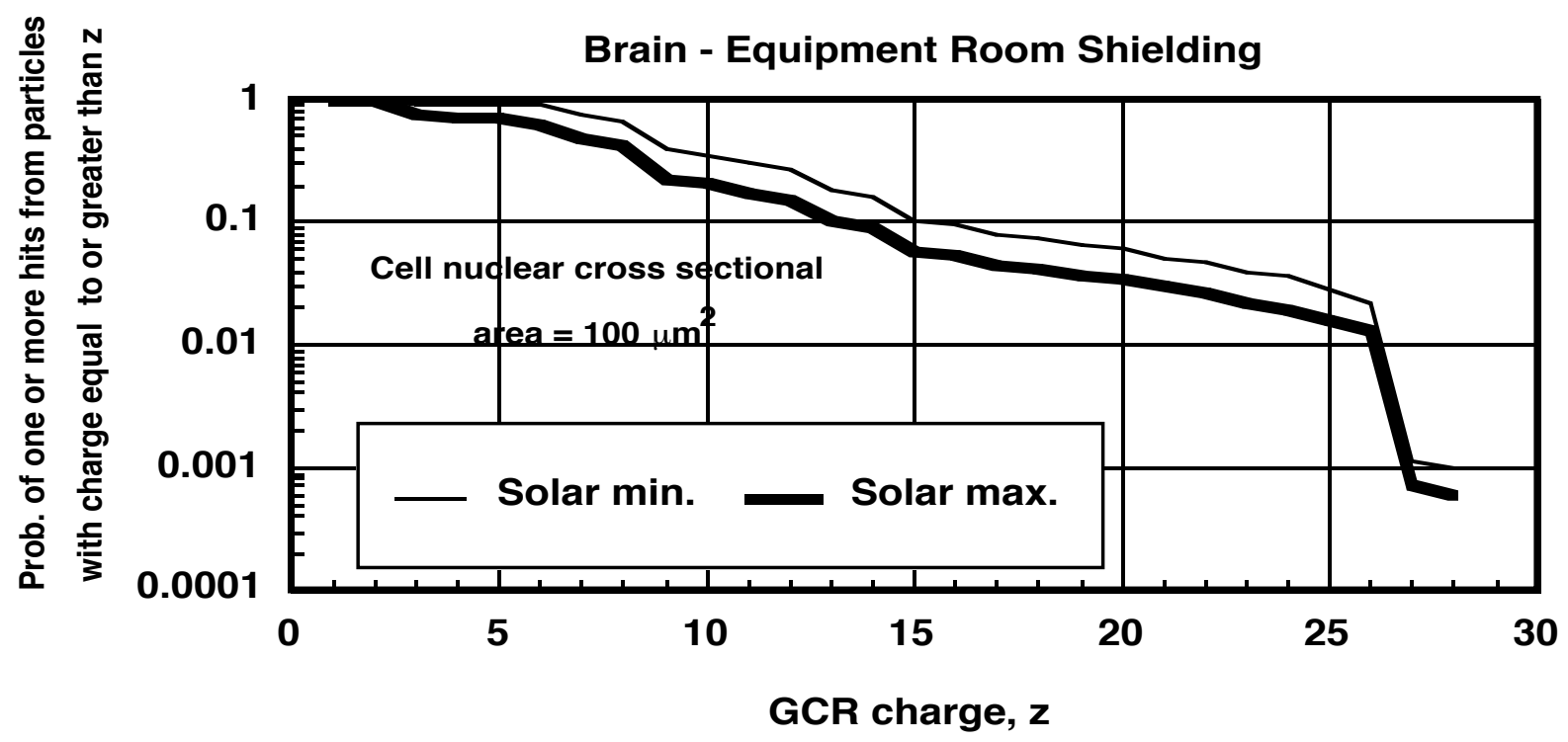

Fig. 4. Comparison of the probabilities of one or more direct hits by particles of solar maximum (thick line) and solar minimum (thin line) spectra with charge greater than or equal to $z$ plotted against $z$ for cell nuclei of the thalamus and nucleus basalis of Meynert $\left(100 \mu \mathrm{m}^{2}\right.$ area) in the brain of a space traveler inside the "equipment room" shielding configuration on a three-year Mars mission. 
Table 1. Probabilities of Hitting Sensitive Sites on a Three-year Mars Mission

\begin{tabular}{|c|c|c|c|c|c|c|c|c|}
\hline & \multicolumn{4}{|c|}{ By particles with $z \geq 15$} & \multicolumn{4}{|c|}{ By iron ions $(z=26)$} \\
\hline & $\begin{array}{r}\text { Pres } \\
\text { ve } \\
\end{array}$ & $\begin{array}{l}\text { Irized } \\
\text { sel } \\
\end{array}$ & $\begin{array}{r}\text { Equ } \\
1 \\
\end{array}$ & $\begin{array}{l}\text { ment } \\
\text { m }\end{array}$ & $\begin{array}{r}\text { Pres } \\
\text { ve } \\
\end{array}$ & $\begin{array}{l}\text { rized } \\
\text { el }\end{array}$ & $\begin{array}{r}\text { Equi } \\
\text { ro } \\
\end{array}$ & $\begin{array}{l}\text { ment } \\
\text { m }\end{array}$ \\
\hline $\begin{array}{l}\frac{\text { Retinal cells }}{\text { Midget foveal ganglion cells }} \\
\text { Cells }\left(180 \mu \mathrm{m}^{2}\right)^{\mathrm{a}} \\
\text { Nuclei }\left(40 \mu \mathrm{m}^{2}\right)^{\mathrm{a}}\end{array}$ & $\begin{array}{c}\text { Solar } \\
\text { max. } \\
0.13 \\
0.031\end{array}$ & $\begin{array}{c}\text { Solar } \\
\text { min. } \\
0.24 \\
0.059\end{array}$ & $\begin{array}{c}\text { Solar } \\
\text { max. } \\
0.11 \\
0.026\end{array}$ & $\begin{array}{c}\text { Solar } \\
\text { min. } \\
0.20 \\
0.048\end{array}$ & $\begin{array}{l}\text { Solar } \\
\text { max. } \\
0.038 \\
0.0086\end{array}$ & $\begin{array}{l}\text { Solar } \\
\text { min. } \\
0.071 \\
0.016\end{array}$ & $\begin{array}{c}\text { Solar } \\
\text { max. } \\
0.030 \\
0.0067\end{array}$ & $\begin{array}{l}\text { Solar } \\
\text { min. } \\
0.053 \\
0.012\end{array}$ \\
\hline $\begin{array}{l}\text { Brain neural cells \& nuclei } \\
\text { Hippocampal cell nuclei } \\
\quad\left(60 \mu \mathrm{m}^{2}\right)^{\mathrm{b}}\end{array}$ & 0.041 & 0.076 & 0.035 & 0.062 & 0.010 & 0.018 & 0.0079 & 0.014 \\
\hline $\begin{array}{l}\text { Thalamus and nucleus of } \\
\text { basalis of Meynert cell nuclei } \\
\qquad\left(100 \mu \mathrm{m}^{2}\right)^{\mathrm{c}, \mathrm{d}}\end{array}$ & 0.067 & 0.12 & 0.057 & 0.10 & 0.017 & 0.030 & 0.013 & 0.023 \\
\hline Average neural cell $\left(471 \mu \mathrm{m}^{2}\right)^{\mathrm{e}}$ & 0.28 & 0.46 & 0.24 & 0.40 & 0.076 & 0.13 & 0.060 & 0.10 \\
\hline
\end{tabular}

${ }^{\mathrm{a}}$ Dacey (1993); ${ }^{\mathrm{b}}$ Dani et al. (1994); ${ }^{\mathrm{C}}$ Pakkenberg and Gundersen (1988); ${ }^{\mathrm{d}}$ LaCalle et al. (1991); ${ }^{\mathrm{e}}$ calculated from

Mani et al. (1986), Pakkenberg and Gundersen (1988) and LaCalle et al. (1991).

\section{DISCUSSION}

From Table 1, we note that the probability of hits at solar maximum varies from between $50 \%$ and $60 \%$ of the probability of hits at solar minimum, depending on the shielding configuration chosen and whether particles with $z \geq 15$ or iron ions $(z=26)$ are considered. From Figure 4 we see that this percentage reduction appears constant at high GCR charges and diminishes at lower values of the charge, i.e., where the probability of a hit approaches unity.

If it is known how many cells are in the organ of interest, the total number of cells or cell nuclei hit can be calculated simply by multiplying the probability of hitting one cell or cell nucleus by the total number of cells. The total number of cells in the macula has been estimated to be $6.5 \times 10^{4}$ (Curcio and Allen, 1990, Gao and Hollyfield, 1992), in the hippocampus, $4.32 \times 10^{7}$ (West, 1993), in the nucleus basilis of Meynert, 4116 (Dani et al., 1994) and in the thalamus $1.83 \times 10^{6}$ (Pakkenberg and Gundersen, 1988). Comparison of the results for solar maximum with the previous results for solar minimum, give the same percentage reduction factors as seen above. Thus, for example, 6 million out of 43 million hippocampal cells hit at solar minimum would be reduced to about 3.3 million hit by one or more iron ions inside the pressure vessel on a three year mission at solar maximum, and of 1.83 million thalamus nuclei, 55 thousand hit by one or more iron ions at solar minimum would be reduced to 31 thousand hit at solar maximum.

\section{CONCLUSION}

We conclude that although any risk to the central nervous system caused by the heavy component of the galactic cosmic rays will be less at solar maximum than at solar minimum, it will be diminished by less than a factor of two, and this does not depend on the charge of the particles considered, at least for charges with $z=$ 15 or greater. Because the risk of large solar particle events is considerably greater during the solar active period of the solar cycle and the decrease in potential risk from the heavy component of the galactic cosmic rays is modest (less than a factor of two), the solar modulation of galactic cosmic ray fluences is not a compelling reason to avoid solar minimum for a manned mission to Mars.

\section{ACKNOWLEDGMENTS}

This work was supported in part by the Office of Space Radiation Health, NASA Headquarters, Washington, DC. 


\section{REFERENCES}

Billings M. P. and W. R. Yucker, Summary final report. The Computerized Anatomical Man (CAM) Model, Report MDC-G4655, McDonnell Douglas Company, Huntington Beach, CA, 1973.

Cucinotta, F. A. , L. W. Townsend, J. W Wilson, J. L Shinn,G. D. Badhwar, and R. R. Dubey, Light ion component of the galactic cosmic rays: Nuclear interactions and transport theory. Adv. in Space Res., 17:77-86; 1995.

Curcio, C. A., and K. A. Allen, Topography of ganglion cells in human retina, J. Comp. Neurol. 300, 5 (1990).

Curtis, S. B. Frequencies of heavy ions in space and their biologically important characteristics, Life Sciences and Space Research XI, Proc. of the Fifteenth Plenary Meeting of COSPAR, Akademie - Verlag, Berlin, 209-214 (1973).

Curtis, S. B., M. E. Vazquez, J.W. Wilson, W. Atwell, M. Kim, and J. Capala, Cosmic ray hit frequencies in critical sites in the central nervous system, Adv. Space Sci., (1998).

Dacey, D. M., The mosaic of midget ganglion cells in the human retina, J. Neurosci. 13(12), 5334 (1993).

Dani, S. U., J. E. H. Pitella, A. Hori, B. Bergman, A. C. Stan, and G. F. Walter, Different rates of neuronal degeneration: An exquisite variation of the "cascade" hypothesis, Dementia, 5, 110 (1994).

Gao, H., and J. G. Hollyfield, Aging of the human retina, Invest. Ophthalmol. Vis. Sci. 33, 1 (1992).

Gauger, G. E., C. A. Tobias, T. Yang, and M. Whitney, The effects of space radiation of the nervous system, Adv. Space Res. 6(11), 243 (1986).

Grahn, D., ed., HZE-Particle Effects in Manned Spaceflight, National Academy of Sciences, Washington, D.C., 1973.

de Lacalle, S. I. Iraizoz, and L. M. Gonzalo, Differential changes in cell size and number in topograghic subdivisions of human basal nucleus in normal aging, Neuroscience 43(2/3), 445 (1991).

Mani, R. B., J. B. Lohr, and D. V. Jeste, Hippocamnpal pyramidal cells and aging in the humans: A quantitative study of neuronal loss in sectors CA1 and CA4, Exp. Neurol., 94, 29 (1986).

National Council of Radiation Protection and Measurements, Guidance on Radiation Received in Space Activities, NCRP Report No. 89, NCRP, Bethesda, (1989).

Pakkenberg B., and H. J. G. Gundersen, Total number of neurons and glial cells in human brain nuclei estimated by the disector and the fractionator, Journal of Microscopy 150(1), 1 (1988).

Wilson, J. W. and F. M. Denn, Preliminary Analysis of the Implications of Natural Radiations on Geostationary Operations. NASA TN D-8290, 1976.

Wilson, J. W., F. F. Badavi, F. A. Cucinotta, J. L. Shinn, G. D. Badhwar, R. Silberberg, C. H. Tsao, L. W. Townsend, and R. K. Tripathi. HZETRN: Description of a Free-Space Ion and Nucleon Transport and Shielding Computer Program. NASA TP 3495, 1995. 